\title{
Properties of Intersecting p-branes in Various Dimensions
}

\author{
I. Ya. Aref'eva* \\ Steklov Mathematical Institute, Gubkin 8, GSP-1, 117966, Moscow, Russia \\ M. G. Ivanov ${ }^{\dagger}$ \\ Department of General and Applied Physics, Moscow Institute of Physics and Technology, \\ Instituski per., 9, Dolgoprudnyi, Moscow region, Russia \\ O. A. Rytchkov \\ Department of Theoretical Physics, Moscow State University, \\ Moscow 119 899, Russia
}

(June 27, 2018)

\begin{abstract}
General properties of intersecting extremal p-brane solutions of gravity coupled with dilatons and several different d-form fields in arbitrary space-time dimensions are considered. It is shown that heiristically expected properties of the intersecting p-branes follow from the explicit formulae for solutions. In particular, harmonic superposition and $S$-duality hold for all p-brane solutions. Generalized $T$-duality takes place under additional restrictions on the initial theory parameters.
\end{abstract}

\section{INTRODUCTION}

There has been recently considerable progress in the study of classical p-brane solutions (for review see [1] and references therein) of higher dimensional gravity coupled with matter. p-brane solutions in 10 and 11 dimensions play a key role for probing the duality conjectures [2 [6] which relate five known superstrings and M-theory. A study of p-brane intersections is a subject of growing interest because when enough branes intersect one gets, as a rule, solutions with a regular horizon. The microscopic interpretation of the Bekenstein-Hawking entropy within string theory [7] has also stimulated investigations of the intersecting (composite) p-brane solutions. Several composite p-brane solutions in $D=10$ and $D=11$ have been obtained [8 25].

Heuristic scheme of constructing of p-brane intersections was based on string theory representation of the branes, duality and supersymmetry. This scheme involves the harmonic function superposition rule for the intersecting pbranes. This rule was formulated [11] in $D=11$ and $D=10$. Using T-duality and the supersymmetry requirements intersections of p-branes (more exactly M-branes and D-branes) have been recently classified in [26]. In 27] it was shown, that the intersection rules applying to type II string theories and M-theory are consistent with the picture that open brane can have boundaries on some other branes.

Other approach to the problem was elaborated in the papers [28 36]. In these papers p-brane intersection rules were found from the equations of motion. The aim of this paper is to summarize these results, and to show that heiristically expected properties of the intersecting p-branes follow from the explicit formulae for solutions.

The paper is organized as follows. In Section 2 we remind single p-brane solutions and introduce graphic representations for them. In Section 3 the main steps of finding p-brane solutions are sketched and general composite p-brane solutions are presented. In Section 4 we collect the formulae for the entropy and ADM mass. In the section 5 we discuss $S$-duality, which is a specific property of our solutions. Harmonic function rule is generalized on arbitrary space-time dimensions in Section 6. T-duality transformations are considered in Section 7. In Section 8 we modify our results for the case of an arbitrary space-time signature. In Section 9 we analyze supersymmetry in the special case of 11D supergravity.

\footnotetext{
*e-mail: arefeva@arevol.mian.su

${ }^{\dagger}$ e-mail: mgi@landau.ac.ru

${ }^{\ddagger}$ e-mail: rytchkov@grg1.phys.msu.su
} 


\section{SINGLE P-BRANE SOLUTIONS}

Let us consider the theory with the following action

$$
I=\frac{1}{2 \kappa^{2}} \int d^{D} X \sqrt{-g}\left(R-\frac{1}{2}(\nabla \phi)^{2}-\frac{e^{-\alpha \phi}}{2(d+1) !} F_{d+1}^{2}\right),
$$

where $F_{d+1}$ is a $d+1$ differential form, $F_{d+1}=d A_{d}, \phi$ is a dilaton.

This action admits the elementary p-brane solution 37

$$
\begin{gathered}
d s^{2}=H^{\tau}(x) \sum_{\mu, \nu=0}^{d-1} \eta_{\mu \nu} d y^{\mu} d y^{\nu}+H^{\rho}(x) \sum_{\gamma=1}^{D-d} d x^{\gamma} d x^{\gamma}, \\
A_{d}=2 \Delta^{-1 / 2} H^{-1}(x) d y^{0} \wedge \ldots \wedge d y^{d-1},
\end{gathered}
$$

where $H(x)$ is a harmonic function. Solution (2), (3) generalizes the well-known Majumdar-Papapetrou solutions [38]. The exponents are defined by the parameters of the theory

$$
\tau=-\frac{4(D-2-d)}{\Delta(D-2)}, \quad \rho=\frac{4 d}{\Delta(D-2)}
$$

where we use the standard notation

$$
\Delta=\alpha^{2}+\frac{2 d(D-2-d)}{D-2} .
$$

In the p-brane terminology [1] the solution (2)-(5) describes an electrically charged p-brane, where $y$-coordinates correspond to the worldvolume directions and $x$-coordinates to directions transverse to the brane. It is convenient to represent every $y^{k}$-coordinate by " $\times$ " and every $x$-coordinate by "-" [26]. One has the following representation of the metric

$$
d s^{2}=\underbrace{\times \times \cdots \times}_{d} \underbrace{--\cdots-}_{D-d} .
$$

It is also convenient to present the gauge field (3) as a row with d circles "o"

$$
\begin{array}{|l|l|}
\hline \circ \circ \cdots \circ \\
\hline
\end{array}
$$

Fig.1

where the circles correspond to indices of the non-zero component.

The action (1) admits also a solitonic p-brane solution [39]

$$
\begin{gathered}
d s^{2}=U^{-\rho}(x) \sum_{\mu, \nu=0}^{D-d-3} \eta_{\mu \nu} d y^{\mu} d y^{\nu}+U^{-\tau}(x) \sum_{\gamma=1}^{d+2} d x^{\gamma} d x^{\gamma}, \\
F=2 \Delta^{-1 / 2} * d U,
\end{gathered}
$$

where $*$ is a Hodge dual on $\mathbb{R}^{d+2}, U(x)$ is a harmonic function. Using the p-brane interpretation one says that the solution (7), (8) describes the magnetically charged p-brane lying in the $y$-directions. One can present the metric in the following way

$$
d s^{2}=\underbrace{* * \cdots *}_{D-d-2} \underbrace{--\cdots-}_{d+2}
$$

where "*" denote the worldvolume directions. Note that we use the symbol " $\times$ " for the electric p-brane and the symbol "*" for the magnetic one.

For the gauge field we have a different picture with $d+2$ circles "•" 
- $\cdots$

Fig.2

where by "•" we denote subspace on which a Hodge dual acts. For the more complicated ansatzes we have pictures with more then one rows. For example,

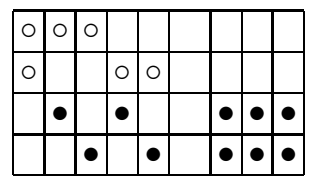

Fig.3

We will call such pictures for the antisymmetric field as incidence tables. In the p-brane terminology the corresponding metric could be presented as

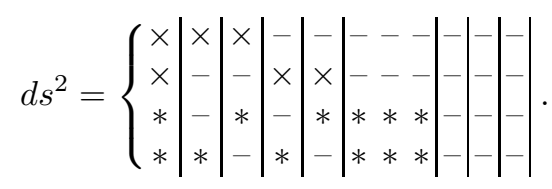

We will call such pictures for the metric as brane incidence tables. The intersections of the p-branes are not arbitrary and governed by characteristic equations which are considered below.

\section{COMPOSITE P-BRANE SOLUTIONS}

The method 28 30,32] of finding the intersecting p-brane solutions involves direct solving the equations of motion for the theory (11). The method consists of the following steps.

Step 1. We assume the metric in the special form (see (11)). In order to simplify the Ricci tensor we also assume that the form of the metric satisfies the Fock-De Donder gauge condition (see (13) below).

Step 2. In order to describe an ansatz for the antisymmetric field we introduce the incidence matrices (see (16), (17)).

Step 3. Using assumed form for the antisymmetric field we calculate components of the energy-momentum tensor. They could be crucially simplified if one assumes so-called "no-force" conditions. Their origin is simple: they eliminate exponents from the components of the energy-momentum tensor.

Step 4. From Maxwell's equations and Bianchi identity we conclude that scalar functions specifying our ansatzes should be harmonic ones.

Step 5. We check that Einstein equations and equation of motion for the dilaton are fulfilled if the incidence matrices satisfy characteristic equations (see (30), (31), (32)).

Let us briefly demonstrate the realization of this program. We assume the metric in the following form

$$
d s^{2}=e^{2 A(x)} \sum_{\mu, \nu=0}^{q-1} \eta_{\mu \nu} d y^{\mu} d y^{\nu}+\sum_{i=q}^{D-s-3} e^{2 F_{i}(x)} d y^{i} d y^{i}+e^{2 B(x)} \sum_{\gamma=D-s-2}^{D-1} d x^{\gamma} d x^{\gamma},
$$

where $\eta_{\mu \nu}$ is a flat Minkowski metric, $A, B$ and $F_{i}$ are functions of $x$. Using the notations $F_{\mu}(x)=A(x)$ and $F_{\gamma}(x)=B(x)$ the above metric may be rewritten as

$$
d s^{2}=\sum_{L=0}^{D-1} e^{2 F_{L}(x)} \eta_{K L} d X^{K} d X^{L} .
$$

If we assume the Fock-De Donder gauge condition, which for metric (11) takes the form

$$
\sum_{L=0}^{D-1} F_{L}-2 B=0
$$


then the Ricci tensor has the following components

$$
R_{K L}=-\sum_{N=0}^{D-1} \partial_{K} F_{N} \partial_{L} F_{N}+2 \partial_{K} B \partial_{L} B-e^{2 F_{L}-2 B} \eta_{K L} \triangle F_{L}
$$

For the d-form $A_{d}$ we consider a class of ansatzes corresponding to $E$ electric and $M$ magnetic charges,

$$
F=\sum_{a}^{E} d A_{a}^{(\mathcal{E})}+\sum_{b}^{M} F_{b}^{(\mathcal{M})}
$$

We will refer to the different terms in (15) as to the different branches of electric and magnetic fields.

To describe electric and magnetic configurations $A_{a}^{(\mathcal{E})}$ and $F_{b}^{(\mathcal{M})}$ we introduce electric and magnetic incidence matrices

$$
\begin{aligned}
\Delta=\left(\Delta_{a L}\right), \quad a=1, \ldots, E, \quad L=0, \ldots, D-1, \\
\Lambda=\left(\Lambda_{b L}\right), \quad b=1, \ldots, M, \quad L=0, \ldots, D-1,
\end{aligned}
$$

respectively. Their rows correspond to independent branches of the electric (magnetic) gauge field and columns refer to the space-time indices. The entries of the incidence matrices are equal to 1 or 0 . Furthermore the electric (magnetic) incidence matrix has an equal number of units in each row, and there are no rows which coincide. Here we don't consider Euclidean p-branes so we assume $\Delta_{a 0}=1, \Delta_{a \alpha}=0, \Lambda_{b 0}=0 \Lambda_{b \alpha}=1$ for all $a$ and $b$ (see section VIII). In a graphic representation we draw " $\circ$ " (for the electric incidence matrix) and "•" (for the magnetic incidence matrix) instead of 1 . Empty space denotes 0 in both cases. Fig.1 and Fig.2 are nothing but a graphic representation of the incidence matrices for the single elementary and solitonic p-brane solutions. The incidence table Fig.3 is an example representing electric and magnetic incidence matrices in a more complicated case.

In the terms of the incidence matrices an electric field is assumed to have the form

$$
A_{a}^{(\mathcal{E})}=h_{a} e^{C_{a}(x)} \bigwedge_{\left\{L \mid \Delta_{a L}=1\right\}} d X^{L},
$$

where we use the notation $\bigwedge_{i=1}^{n} d X^{i}=d X^{1} \wedge \cdots \wedge d X^{n}$.

In the terms of the incidence matrices a magnetic field is assumed in the form

$$
F_{b}^{(\mathcal{M}) K_{1} \ldots K_{d+1}}=\frac{1}{\sqrt{-g}} v_{b} e^{\alpha \phi} \epsilon^{K_{1} \ldots K_{d+1} \beta} \partial_{\beta} e^{\chi}
$$

where we take $K_{i}$ such that $\Lambda_{b K_{i}}=1$.

We will use Einstein equations in the form $R_{K L}=G_{K L}$, there $G_{K L}$ is related with the stress-energy tensor $T_{K L}$ as

$$
G_{K L}=T_{K L}-\frac{g_{K L}}{D-2} T_{P}^{P}
$$

For the considered ansatz the tensor $G$ is

$$
\begin{gathered}
G_{K L}=\frac{1}{2} \partial_{K} \phi \partial_{L} \phi \\
+\sum_{a=1}^{E} \frac{h_{a}^{2}}{2} e^{2 F_{L}-2 B+\mathcal{F}_{a}}\left(-\partial_{K} C_{a} \partial_{L} C_{a}-\eta_{K L}\left\{\Delta_{a L}-\frac{d}{D-2}\right\}\left(\partial C_{a}\right)^{2}\right) \\
+\sum_{b=1}^{M} \frac{v_{b}^{2}}{2} e^{2 F_{L}-2 B+\mathcal{F}_{b}}\left(-\partial_{K} \chi_{b} \partial_{L} \chi_{b}+\eta_{K L}\left\{\Lambda_{b L}-\frac{d}{D-2}\right\}\left(\partial \chi_{b}\right)^{2}\right),
\end{gathered}
$$

where

$$
\begin{aligned}
& \mathcal{F}_{a}=-\alpha \phi-2 \sum_{N=0}^{D-1} \Delta_{a N} F_{N}+2 C_{a} \\
& \mathcal{F}_{b}=\alpha \phi+2 \sum_{N=0}^{D-1} \Lambda_{b N} F_{N}-4 B+2 \chi_{b} .
\end{aligned}
$$

In order to guarantee the above form of the $G$-tensor (the absence of the $(i j)-,(\mu \nu)-,(\mu i)$-, $(\mu \alpha)$ - and $(i \alpha)$-components, where $i \neq j, \mu \neq \nu)$ we have to impose additional restrictions on the incidence matrices. Namely, each two rows 
assumed to have differences in more then two columns. Furthermore, the difference between each row of the electric incidence matrix and each row of the magnetic incidence matrix have to be in more then four columns.

According to step 3 we assume the following "no-force" conditions

$$
\begin{aligned}
& \mathcal{F}_{a}=0, a=1, \ldots, E, \\
& \mathcal{F}_{b}=0, b=1, \ldots, M .
\end{aligned}
$$

The LHS of these conditions are nothing but exponents which enter in the $G$ tensor. Using (24) the magnetic field (19) could be rewritten in the following form

$$
F_{b}^{(\mathcal{M})}=v_{b} e^{-\chi_{b}(x)} * d \chi_{b}(x) \bigwedge_{\left\{i \mid \Lambda_{b i}=1\right\}} d y^{i}
$$

where $*$ is a Hodge dual on the $x$-subspace.

From Maxwell's equations and from Bianchi identity for $F$ under conditions (23) and (24) we get

$$
\Delta C_{a}=\left(\partial C_{a}\right)^{2}, \quad \Delta \chi_{b}=\left(\partial \chi_{b}\right)^{2},
$$

therefore, $H_{a}=e^{-C_{a}}, a=1, \ldots, E$ and $U_{b}=e^{-\chi_{b}}, b=1, \ldots, M$ are harmonic functions.

In order to solve the Einstein equations and the equation of motion for the dilaton we suppose

$$
\begin{aligned}
F_{L} & =\sum_{a=1}^{E} h_{a}^{2} C_{a}\left(\frac{1}{2} \Delta_{a L}-u\right)-\sum_{b=1}^{M} v_{b}^{2} \chi_{b}\left(\frac{1}{2} \Lambda_{b L}-u\right), \\
\phi & =\frac{\alpha}{2}\left[\sum_{a=1}^{E} h_{a}^{2} C_{a}-\sum_{b=1}^{M} v_{a}^{2} \chi_{b}\right],
\end{aligned}
$$

where

$$
u=\frac{d}{2(D-2)} .
$$

Substituting (27) and (28) in (13) one can check that equation (13) is fulfilled. A substitution of (27) and (28) in the "no force" conditions under assumption of the independence of $C_{a}$ and $\chi_{b}$ gives three types of characteristic equations on the incidence matrices

$$
\begin{aligned}
& \frac{\alpha^{2}}{2}-\frac{d^{2}}{D-2}+\sum_{L=0}^{D-1} \Delta_{a L} \Delta_{a^{\prime} L}=0, \quad a \neq a^{\prime}, \quad a, a^{\prime}=1, \ldots, E, \\
& \frac{\alpha^{2}}{2}-\frac{d^{2}}{D-2}+\sum_{L=0}^{D-1} \Lambda_{b L} \Lambda_{b^{\prime} L}-2=0, b \neq b^{\prime}, \quad b, b^{\prime}=1, \ldots, M, \\
& \frac{\alpha^{2}}{2}-\frac{d^{2}}{D-2}+\sum_{L=0}^{D-1} \Delta_{a L} \Lambda_{b L}=0, \quad a=1, \ldots, E, \quad b=1, \ldots, M,
\end{aligned}
$$

where $h_{a}^{2}=v_{b}^{2}=\sigma$,

$$
\sigma=\frac{1}{d t+\alpha^{2} / 4}, \quad t=\frac{D-d-2}{2(D-2)} .
$$

Note that $\sum_{L=0}^{D-1} \Delta_{a L} \Delta_{a^{\prime} L}$ is a number of common columns of $a$ and $a^{\prime}$ branches in the incidence table. Characteristic equations admit solutions only for quantized values of scalar coupling parameter that is in accordance with [23,28, 30].

Note that if the dilaton is absent, then the p-brane solutions are given by the above formula with $\alpha=0$. Corresponding characteristic equations are very restrictive since they have to be solved for integers and they admit non-trivial solutions only for special $D$. In particular, they are $D=6,10,11,18,20,26$.

One can check that if the incidence matrices satisfy the characteristic equations then the Einstein equations hold. Therefore, the form of the metric which solves the theory is 


$$
\begin{aligned}
& d s^{2}=\left(H_{1} H_{2} \cdots H_{E}\right)^{2 u \sigma}\left(U_{1} U_{2} \cdots U_{M}\right)^{2 t \sigma} \\
& \times\left\{\sum_{K, L=0}^{D-s-3}\left(\prod_{a} H_{a}^{\Delta_{a L}} \prod_{b} U_{b}^{1-\Lambda_{b L}}\right)^{-\sigma} \eta_{K L} d y^{K} d y^{L}+\sum_{\gamma} d x^{\gamma} d x^{\gamma}\right\},
\end{aligned}
$$

where $H_{a}$ and $U_{b}$ are harmonic functions, $u, t$ and $\sigma$ are given by (29) and (33).

The explicit formula for the dilaton has the form

$$
e^{\phi}=\left(\prod_{a} H_{a}^{-1} \prod_{b} U_{b}\right)^{\frac{\alpha \sigma}{2}} .
$$

The electric strength is

$$
F_{\alpha \mu_{1}^{a} \cdots \mu_{d}^{a}}^{(\mathcal{E})}=\mp \sqrt{\sigma} e^{\frac{1}{2} \alpha \phi} E_{\mu_{1}^{a} \cdots \mu_{d}^{a}}^{a} \partial_{\alpha} \ln H_{a},
$$

and the magnetic one is

$$
F_{\mu_{0}^{b} \cdots \mu_{d}^{b}}^{(\mathcal{M})}=\mp \sqrt{\sigma} e^{\frac{1}{2} \alpha \phi} E_{\mu_{0}^{b} \cdots \mu_{d}^{b} \alpha}^{b} g^{\alpha \beta} \partial_{\beta} \ln U_{b},
$$

where $\mu^{a} \in\left\{L \mid \Delta_{a L}=1\right\}, \mu^{b} \in\left\{L \mid \Lambda_{b L}=1\right\}$,

$$
\begin{aligned}
& E_{\mu_{1}^{a} \cdots \mu_{d}^{a}}^{a}=\sqrt{-\prod_{L=0}^{D-1} g_{L L}^{\Delta_{a} L} \epsilon_{\mu_{1}^{a} \cdots \mu_{d}^{a}},} \\
& E_{\mu_{0}^{b} \cdots \mu_{d}^{b} \alpha}^{b}=\sqrt{\prod_{L=0}^{D-1} g_{L L}^{\Lambda_{b L}} \epsilon_{\mu_{0}^{b} \cdots \mu_{d}^{b} \alpha}}
\end{aligned}
$$

are forms of $d$ and $d+2$ dimensional volume.

The approach considered above could be obviously generalized to the actions with $k$ antisymmetric fields and several dilatons

$$
I=\frac{1}{2 \kappa^{2}} \int d^{D} X \sqrt{-g}\left(R-\frac{1}{2}(\nabla \vec{\phi})^{2}-\sum_{I=1}^{k} \frac{e^{-\vec{\alpha}^{(I)} \vec{\phi}}}{2\left(d_{I}+1\right) !} F_{d_{I}+1}^{(I) 2}\right) .
$$

In this case we introduce $2 k$ incidence matrices. Electric and magnetic fields are assumed in the form

$$
\begin{gathered}
A_{a}^{(I)}=h_{a}^{(I)} e^{C_{a}^{(I)}(x)} \bigwedge_{\left\{L \mid \Delta_{a L}^{(I)}=1\right\}} d X^{L}, \\
F_{b}^{(I) K_{1} \ldots K_{d_{I}+1}}=\frac{1}{\sqrt{-g}} v_{b}^{(I)} e^{\vec{\alpha}^{(I)} \vec{\phi}_{\epsilon} \epsilon_{1} \ldots K_{d+1} \beta} \partial_{\beta} e^{\chi^{(I)}},
\end{gathered}
$$

where $I=1, \ldots, k$ and we take $K_{i}$ such that $\Lambda_{b K_{i}}^{(I)}=1$. Instead of (30), (31) and (32) one has the following characteristic equations

$$
\begin{gathered}
\left(1-\delta_{I J} \delta_{a a^{\prime}}\right)\left\{\frac{\vec{\alpha}^{(I)} \vec{\alpha}^{(J)}}{2}-\frac{d_{I} d_{J}}{D-2}+\sum_{L=0}^{D-1} \Delta_{a L}^{(I)} \Delta_{a^{\prime} L}^{(J)}\right\}=0, \\
\left(1-\delta_{I J} \delta_{b b^{\prime}}\right)\left\{\frac{\vec{\alpha}^{(I)} \vec{\alpha}^{(J)}}{2}-\frac{d_{I} d_{J}}{D-2}+\sum_{L=0}^{D-1} \Lambda_{b L}^{(I)} \Lambda_{b^{\prime} L}^{(J)}-2\right\}=0,
\end{gathered}
$$




$$
\frac{\vec{\alpha}^{(I)} \vec{\alpha}^{(J)}}{2}-\frac{d_{I} d_{J}}{D-2}+\sum_{L=0}^{D-1} \Delta_{a L}^{(I)} \Lambda_{b^{\prime} L}^{(J)}=0
$$

The constants $h_{a}^{(I)}$ and $v_{b}^{(I)}$ are given by

$$
h_{a}^{(I)^{2}}=v_{b}^{(I)^{2}}=\sigma^{(I)}, \quad \text { where } \quad \sigma^{(I)}=\frac{1}{t^{(I)} d_{I}+\frac{1}{4} \vec{\alpha}^{(I)}{ }^{2}}, \quad t^{(I)}=\frac{D-2-d_{I}}{2(D-2)} .
$$

Let us present the metric in the form where the overall conformal factor which multiplies the transverse $x$-part is separated

$$
\begin{gathered}
d s^{2}=\prod_{I=1}^{k}\left(H_{1}^{(I)} H_{2}^{(I)} \cdots H_{E_{I}}^{(I)}\right)^{2 u^{(I)} \sigma^{(I)}}\left(U_{1}^{(I)} U_{2}^{(I)} \cdots U_{M_{I}}^{(I)}\right)^{2 t^{(I)} \sigma^{(I)}} \\
\left.\times\left\{\sum_{L=0}^{D-s-3} \prod_{I=1}^{k}\left(\prod_{a} H_{a}^{(I)}{ }_{a L}^{(I)} \prod_{b} U_{b}^{(I)}\right)^{1-\Lambda_{b L}^{(I)}}\right)^{-\sigma^{(I)}} \eta_{K L} d y^{K} d y^{L}+\sum_{\gamma} d x^{\gamma} d x^{\gamma}\right\}
\end{gathered}
$$

where

$$
u^{(I)}=\frac{d_{I}}{2(D-2)} .
$$

Under assumption $s>0$ we take the harmonic functions in the form

$$
H_{a}^{(I)}=1+\sum_{c} \frac{Q_{a c}^{(I)}}{\left|x-x_{a c}\right|^{s}}, \quad U_{b}^{(I)}=1+\sum_{c} \frac{P_{b c}^{(I)}}{\left|x-x_{b c}\right|^{s}} .
$$

The algebraic method [28 30]33] can be also used for finding solutions with depending harmonic functions.

\section{ADM MASS AND AREA OF HORIZON}

The representation (47) is convenient for calculating the ADM mass and the entropy. Let the harmonic functions have the form (49) and all these functions have the same centers $\left(Q_{a c}^{(I)}, P_{b c}^{(I)}>0\right.$ for all $\left.a, b, c, I\right)$.

The ADM mass has the form

$$
M=\frac{L^{d-1} \omega_{s+1} s}{2 \kappa^{2}} \sum_{I} \sigma^{(I)}\left(\sum_{a c} Q_{a c}^{(I)}+\sum_{b c} P_{b c}^{(I)}\right),
$$

where $\omega_{s+1}$ is a volume of the $s+1$-dimensional sphere,

$$
\omega_{s+1}=\frac{2 \pi^{\frac{s+2}{2}}}{\Gamma\left(\frac{s+2}{2}\right)}
$$

and $L$ is a period of all $y_{i}, i=1, \ldots, D-s-3$.

Under the condition

$$
\frac{s}{2} \sum_{I} \sigma^{(I)}\left(E_{I}+M_{I}\right)=s+1
$$

the area of horizon has the form

$$
\mathcal{A}_{D-2}=\omega_{s+1} L^{D-s-3} \sum_{c} \prod_{I}\left(\prod_{a} Q_{a c}^{(I)} \prod_{b} P_{b c}^{(I)}\right)^{\frac{\sigma^{(I)}}{2}},
$$

One can also get non-trivial entropy if $q \geq 2$ and instead of (52) the following condition is assumed 


$$
\frac{s}{2} \sum_{I} \sigma^{(I)}\left(E_{I}+M_{I}\right)=\frac{s}{2}+1 .
$$

To get non-trivial entropy we make a boost

$$
-d y_{0}^{2}+d y_{1}^{2} \longrightarrow d u d v+K(x) d u^{2},
$$

where

$$
u=y_{1}+y_{0}, v=y_{1}-y_{0}, K(x)=\sum_{c} \frac{Q_{c}}{\left|x-x_{c}\right|^{s}}, x_{c}=x_{a c} .
$$

In this case the area of gorizon is given by

$$
\mathcal{A}_{D-2}=\omega_{s+1} L^{D-s-3} \sum_{c} Q_{c}^{\frac{1}{2}} \prod_{I}\left(\prod_{a} Q_{a c}^{(I)} \prod_{b} P_{b c}^{(I)}\right)^{\frac{\sigma^{(I)}}{2}} .
$$

\section{V. $S$-DUALITY}

In order to demonstrate $S$-duality let us consider a new action, which could be obtained from the action (40) by replacing an antisymmetric field $F_{d_{I}}^{(I)}$ by another field $F_{\tilde{d}_{I}}^{(I)}$,

$$
\tilde{d}_{I}=D-2-d_{I},
$$

and changing the signs of the corresponding dilaton coupling constants on the opposite ones:

$$
\tilde{\vec{\alpha}}^{(I)}=-\vec{\alpha}^{(I)} .
$$

$S$-duality transforms the solutions of the theory (40) into the solutions of the theory with a new action. The corresponding transformations of the incidence matrices are

$$
\begin{aligned}
\Delta_{a L}^{(I)} & \rightarrow \tilde{\Delta}_{b L}^{(I)}=1-\Lambda_{b L}^{(I)}, \\
\Lambda_{b L}^{(I)} & \rightarrow \tilde{\Lambda}_{a L}^{(I)}=1-\Delta_{a L}^{(I)} .
\end{aligned}
$$

One can check that the new incidence matrices also satisfy the characteristic equations.

Also one can perform $S$-duality transformation (60)- 61) only for some branches of the fields. In this case the dual theory may have more fields in comparison with the initial one.

$S$-duality becomes evident if we present our results in the p-brane terminology. In order to consider the electric and magnetic p-branes together we introduce the brane incidence matrix $\Upsilon_{P L}$, where $L=0, \ldots, D-1 ; P=1, \ldots, B$, $B$ is a whole number of p-branes. The brane incidence matrix is constructed in the following way

$$
\Upsilon_{P L}=\left(\begin{array}{c}
\Delta_{a L}^{(1)} \\
\Delta_{a L}^{(2)} \\
\vdots \\
\Delta_{a L}^{(k)} \\
1-\Lambda_{b L}^{(1)} \\
1-\Lambda_{b L}^{(2)} \\
\vdots \\
1-\Lambda_{b L}^{(k)}
\end{array}\right) .
$$

The entries of the brane incidence matrix are equal to 1 or 0 . The rows of this matrix correspond to p-branes. Electrically charged p-branes occupy the upper rows of the matrix, magnetically charged p-branes occupy the down rows. The columns of the matrix $\Upsilon_{P L}$ correspond to the space-time indices (similar to $\Delta$ and $\Lambda$ ). This matrix could 
be represented as the brane incidence table (see example (10)). We denote by " $\times$ " electrically charged p-branes and by "*" magnetically charged ones. For $\Upsilon_{P L}$ we have only one characteristic equation instead of three ones. Namely, the equations $(43),(44)$ and $(45)$ in the terms of $\Upsilon_{P L}$ could be rewritten in the following form

$$
\varsigma_{R} \varsigma_{R^{\prime}} \frac{\vec{\alpha}^{(R)} \vec{\alpha}^{\left(R^{\prime}\right)}}{2}-\frac{d_{R} d_{R^{\prime}}}{D-2}+\sum_{L=0}^{D-1} \Upsilon_{R L} \Upsilon_{R^{\prime} L}=0, R \neq R^{\prime}
$$

where $\varsigma_{R}$ is -1 for the electric p-branes and +1 for the magnetic ones, $\vec{\alpha}^{(R)}$ are dilatons coupling constants connected with the $p_{R}$-brane, $d_{R}=p_{R}+1$. This unified form of the characteristic equations is a manifestation of the $S$-duality.

\section{HARMONIC FUNCTION RULE}

Our solution 47 has a very simple structure. This becomes obvious if one rewrites the metric in the following form:

$$
g_{K K}=\prod_{I} \prod_{a} g_{K K}^{(I) a} \prod_{b} g_{K K}^{(I) b}
$$

where

$$
\begin{aligned}
& g_{K K}^{(I) a}=\left(H_{a}^{(I) \Delta_{a K}}\right)^{\tau^{(I)}}\left(H_{a}^{(I)\left(1-\Delta_{a K}\right)}\right)^{\rho^{(I)}}, \\
& g_{K K}^{(I) b}=\left(U_{b}^{(I) \Lambda_{b K}}\right)^{-\tau^{(I)}}\left(U_{b}^{(I)\left(1-\Lambda_{b K}\right)}\right)^{-\rho^{(I)}} .
\end{aligned}
$$

According to 46 ) and $(48)$ the exponents are given by:

$$
\tau^{(I)} \equiv-2 t^{(I)} \sigma^{(I)}=-\frac{4\left(D-2-d_{I}\right)}{\Delta^{(I)}(D-2)}, \quad \rho^{(I)} \equiv 2 u^{(I)} \sigma^{(I)}=\frac{4 d_{I}}{\Delta^{(I)}(D-2)},
$$

where $\Delta^{(I)}$ is a generalization of (5)

$$
\Delta^{(I)}=\vec{\alpha}^{(I) 2}+\frac{2 d_{I}\left(D-2-d_{I}\right)}{D-2} .
$$

For given incidence matrices and values of $\tau^{(I)}$ and $\rho^{(I)}(64)-(66)$ gives the following rule for constructing a metric. For each space-time direction the coefficient in the metric is a product of functions $H_{a}$ and $U_{b}$ in an appropriate power. Namely, we put $H_{n}^{(I)} \tau^{\tau^{(I)}}\left(U_{n}^{(I)}{ }^{-\rho^{(I)}}\right)$ if the corresponding direction belongs to the $n$-th $(d-1)-$ electric $((D-d-3)-$ magnetic) brane, and we put $H_{n}^{(I)} \rho^{\rho^{(I)}}\left(U_{n}^{(I)}{ }^{-\tau^{(I)}}\right)$ if the corresponding direction is transverse to $(d-1)-$ electric $\left((D-d-3)-\right.$ magnetic) brane. Note that $\tau^{(I)}$ and $\rho^{(I)}$ are the same as in the corresponding single brane. Certainly, one has to assume that the incidence matrices satisfy the characteristic equations (43)-(45).

There is another point of view, based on the metric representation (47). The overall conformal factor in (47) can be rewritten as

$$
\begin{aligned}
& \prod_{I=1}^{k}\left(H_{1}^{(I)} H_{2}^{(I)} \cdots H_{E_{I}}^{(I)}\right)^{2 u^{(I)} \sigma^{(I)}}\left(U_{1}^{(I)} U_{2}^{(I)} \cdots U_{M_{I}}^{(I)}\right)^{2 t^{(I)} \sigma^{(I)}} \\
= & \prod_{I=1}^{k}\left(H_{1}^{(I)} H_{2}^{(I)} \cdots H_{E_{I}}^{(I)}\right)^{\frac{d_{I}}{D-2} \sigma^{(I)}}\left(U_{1}^{(I)} U_{2}^{(I)} \cdots U_{M_{I}}^{(I)}\right)^{\frac{D-d_{I}-2}{D-2} \sigma^{(I)}} .
\end{aligned}
$$

Since the magnetic p-brane is connected with the $(D-p-3)$-form, the conformal factor could be unified

$$
\prod_{R=1}^{B} H_{R}^{\frac{p_{R}+1}{D-2} \sigma^{(R)}}
$$

where we have a product of all harmonic functions in an appropriate power which is independent on the p-brane charge and is determined by the p-brane dimension. For each space-time dimension the coefficient in the metric (in 
brackets) is also a product of harmonic functions in power $\sigma^{(R)}$. Namely, we put $H_{R}^{-\sigma^{(R)}}$ if the corresponding direction belongs to the $R$-th brane. So the form of the metric doesn't depend on the type of p-brane charges and could be constructed in terms of the brane incidence matrix (which describes p-brane intersections only)

$$
d s^{2}=\prod_{R=1}^{B} H_{R}^{\frac{p_{R}+1}{D-2} \sigma^{(R)}}\left\{\sum_{L=0}^{D-s-3}\left(\prod_{R=1}^{B} H_{R}^{\Upsilon_{R L}}\right)^{-\sigma^{(R)}} \eta_{K L} d y^{K} d y^{L}+\sum_{\gamma} d x^{\gamma} d x^{\gamma}\right\} .
$$

Therefore the formula (81) gives a $D$-dimensional generalization of the "harmonic function rule" found before for $D=10,11$ [1].

\section{T-DUALITY}

Let us consider generalized $T$-duality transformations. $T$-duality transforms solutions for the action (40) with one set of fields into solutions of the action (40) with another set of fields. We perform $T$-duality transformation along the direction corresponding to $y_{i_{0}}$ coordinate, $q \leq i_{0} \leq D-s-3$. T-duality acts on the brane incidence matrix $\Upsilon_{R L}$ as follows. We select the $i_{0}$-th column, change 1 into 0 and vice versa and obtain a new brane incidence matrix. This matrix satisfies the characteristic equation if we simultaneously change dilaton coupling constants. More precisely, new dilaton coupling constants $\vec{\beta}_{R}$ are connected with the old ones $\vec{\alpha}_{R}$ in the following way

$$
\begin{aligned}
\frac{\vec{\beta}_{R} \vec{\beta}_{R^{\prime}}}{2}=\frac{\vec{\alpha}_{R} \vec{\alpha}_{R^{\prime}}}{2}- & 1+\Upsilon_{R i_{0}}+\Upsilon_{R^{\prime} i_{0}}+\frac{1}{D-2}\left[\left(1-2 \Upsilon_{R i_{0}}\right)\left(1-2 \Upsilon_{R^{\prime} i_{0}}\right)\right. \\
& \left.+\left(1-2 \Upsilon_{R i_{0}}\right) d_{R^{\prime}}+\left(1-2 \Upsilon_{R^{\prime} i_{0}}\right) d_{R}\right] .
\end{aligned}
$$

In particular cases these relations give rather restrictive conditions on the initial theory parameters. For example, let us consider the case of one dilaton and one antisymmetric field and let us deal with the electrically charged p-branes. We make the mapping

$$
I(D, d, \alpha) \longmapsto I\left(D, d-1, d+1, \alpha_{1}, \alpha_{2}\right)
$$

and call it as a generalized $T$-duality transformation. $T$-duality [2, 6 in in the case of IIA superstring transforms solutions with non-zero 2-form into solutions with non-zero 1- and 3-forms [26]. After performing $T$-duality we have a new brane incidence matrix $\Upsilon_{R L}^{\prime}$ which is effectively a composition of the brane incidence matrices for $(d-2)$ - and $d$-branes. In the case $E^{\prime}>1, E^{\prime \prime}>1$ the characteristic equations (72) are consistent only if

$$
\alpha^{2}=\frac{(2 d-D+2)^{2}}{2(D-2)}
$$

(for $E^{\prime}=1$ or $E^{\prime \prime}=1$ the characteristic equations are less restrictive). Only under condition (74) $T$-duality in the form (73) takes place. Using (34) and (47) one can check that the values of $\sigma$ which specify the relative transverse components of the metrics corresponding to the theories related by $T$-duality are the same.

\section{THE CASE OF ARBITRARY SIGNATURE}

p-brane solutions also exist in the case of an arbitrary space-time signature: $\eta=\operatorname{diag}( \pm 1, \ldots, \pm 1)$. In this case one deals with a modified action

$$
I=\frac{1}{2 \kappa^{2}} \int d^{D} X \sqrt{|g|}\left(R-\frac{1}{2}(\nabla \vec{\phi})^{2}-\sum_{I=1}^{k} \frac{s_{I} e^{-\vec{\alpha}^{(I)} \vec{\phi}}}{2\left(d_{I}+1\right) !} F_{d_{I}+1}^{(I) 2}\right),
$$

where $s_{I}= \pm 1$. Instead of old restrictions for the incidence matrices one has new ones

$$
\begin{array}{lll}
s_{I} \prod_{L=0}^{D-1}\left(\eta_{L L}\right)^{\Delta_{a L}^{(I)}}=-1 & \text { instead of } & \Delta_{a 0}^{(I)}=1, \\
s_{I} \prod_{L=0}^{D-1}\left(\eta_{L L}\right)^{\Lambda_{b L}^{(I)}}=+1 & \text { instead of } & \Lambda_{b 0}^{(I)}=0 .
\end{array}
$$


In the unified notation we may rewrite this restrictions as

$$
s_{I} \prod_{L=0}^{D-1}\left(\eta_{L L}\right)^{\Delta_{R L}}=\varsigma_{R}
$$

where $\Delta_{R L}$ is the matter incidence matrix, which contain rows corresponding to both types of p-branes, electric and magnetic. The characteristic equations remain the same.

Under the $S$-duality transformation in addition to $(58),(59)$ one has also to perform a change

$$
s_{I} \rightarrow \tilde{s}_{I}=-\operatorname{det}\left(\eta_{K L}\right) s_{I}
$$

\section{SUPERSYMMETRY IN 11D SUPERGRAVITY}

Let us recall that some p-brane solutions were obtained using requirements of supersymmetry. Known supersymmetric p-brane solutions admit an existence of Killing spinors. In this Section we are going to examine relations between the problem of finding Killing spinors and our scheme of finding solutions taking as example 11D supergravity. The bosonic sector of $D=11$ supergravity consists of a metric and a three-form potential. Killing spinors $\varepsilon$ satisfy the following equations

$$
\begin{aligned}
\tilde{D}_{L} \varepsilon & =0 \\
\tilde{D}_{L} & =\partial_{L}+\frac{1}{4} \omega_{L}{ }^{A B} \Gamma_{A B}-\frac{1}{288}\left(\Gamma_{L}^{P Q R S}+8 \Gamma^{P Q R} \delta_{L}^{S}\right) F_{P Q R S},
\end{aligned}
$$

where $P, Q, R, S, L$ are $11 \mathrm{D}$ world indices. $\Gamma_{A}$ are the $11 \mathrm{D}$ Dirac matrices

$$
\begin{array}{r}
\left\{\Gamma_{A}, \Gamma_{B}\right\}=2 \eta_{A B}, \\
\Gamma_{A B \cdots C}=\Gamma_{[A} \Gamma_{B} \ldots \Gamma_{C]} .
\end{array}
$$

Indices $A, B, C$ are $11 \mathrm{D}$ vielbein indices, related with the orthonormal vielbein.

If we assume the ansatz for the metric (11) and for the matter field (18), (19), then in the Fock-De Donder gauge the covariant derivative is

$$
\begin{aligned}
& \tilde{D}_{L}=\partial_{L}-\frac{1}{2} \Gamma_{L}{ }^{K} \partial_{K} F_{L}+\sum_{a} \frac{h_{a}}{6} e^{\mathcal{F}_{a} / 2} \partial_{L} C_{a} \Gamma(a)-\sum_{b} \frac{v_{b}}{12} e^{\mathcal{F}_{b} / 2} \partial_{L} \chi_{b} \Gamma(b) \\
& +\Gamma_{L}{ }^{K} \sum_{a} \frac{h_{a}}{2}\left\{\Delta_{a L}-\frac{1}{3}\right\} e^{\mathcal{F}_{a} / 2} \partial_{K} C_{a} \frac{\Gamma(a)}{2}+\Gamma_{L}{ }^{K} \sum_{b} \frac{v_{b}}{2}\left\{\Lambda_{b L}-\frac{1}{3}\right\} e^{\mathcal{F}_{b} / 2} \partial_{K} \chi_{b} \frac{\Gamma(b)}{2},
\end{aligned}
$$

where

$$
\begin{aligned}
\Gamma(a)=\frac{1}{3 !} E^{\mu_{1}^{a} \mu_{2}^{a} \mu_{3}^{a}} \Gamma_{\mu_{1}^{a} \mu_{2}^{a} \mu_{3}^{a}} & =\prod_{\left\{A \mid \Delta_{a A}=1\right\}} \Gamma_{A}, \\
\Gamma(b)=\frac{1}{5 !} E^{\mu_{1}^{b} \mu_{2}^{b} \mu_{3}^{b} \mu_{4}^{b} \mu_{5}^{b}} \Gamma_{\mu_{1}^{b} \mu_{2}^{b} \mu_{3}^{b} \mu_{4}^{b} \mu_{5}^{b}} & =\prod_{\left\{A \mid \Lambda_{b A}=1\right\}} \Gamma_{A} .
\end{aligned}
$$

Here notations are the same as in (36), (37). For definition of $\mathcal{F}_{a}$ and $\mathcal{F}_{b}$ see (22).

To analyze the consequences of the supersymmetry it is convenient to rewrite the covariant derivative as

$$
\begin{aligned}
\tilde{D}_{L} & =\partial_{L}-\frac{1}{2} \Gamma_{L}{ }^{K} \partial_{K} F_{L}+\sum_{R} \frac{1-3 \varsigma_{R}}{24} h_{R} e^{\mathcal{F}_{R} / 2} \partial_{L} C_{R} \Gamma(R) \\
& +\Gamma_{L}{ }^{K} \sum_{R} \frac{h_{R}}{2}\left\{\Delta_{R L}-\frac{1}{3}\right\} e^{\mathcal{F}_{R} / 2} \partial_{K} C_{R} \frac{\Gamma(R)}{2}
\end{aligned}
$$

The notations in (87) are obvious

$$
C_{R}=\left\{\begin{array}{ll}
C_{a}, & R=a \\
\chi_{b}, & R=b
\end{array}, \quad h_{R}=\left\{\begin{array}{ll}
h_{a}, & R=a \\
v_{b}, & R=b
\end{array} .\right.\right.
$$


Definition of the matrix $\Delta_{R L}$ one can find after equation (78). Note that in the expression (87) for the covariant derivative we do not assume that the metric solves the Einstein equations.

To construct the Killing spinor it is natural to assume that all $\mathcal{F}_{R}$ vanish. These conditions coincide with "no force" conditions (23), (24).

Now we can see that if we assume that there exist spinors $\varepsilon_{0}$ such that

$$
\Gamma_{L}{ }^{K}\left[-\frac{1}{2} \partial_{K} F_{L}+\sum_{R} \frac{h_{R}}{2}\left\{\Delta_{R L}-\frac{1}{3}\right\} \partial_{K} C_{R} \frac{\Gamma(R)}{2}\right] \varepsilon_{0}=0
$$

then the Killing spinors can be found in the form

$$
\varepsilon=\varepsilon_{0} f(x),
$$

where a scalar function $f(x)$ solves the following equations

$$
\left[\partial_{L}+\sum_{R} \frac{1-3 \varsigma_{R}}{24} h_{R} \partial_{L} C_{R} \Gamma(R)\right] \varepsilon_{0} f(x)=0 .
$$

In order to solve the equation (88), let us assume that $F_{L}$ are linear combinations of the functions $C_{R}$ (a "relax" harmonic superposition rule), for simplification of our calculation we shall write $F_{L}=-1 / 2 \sum_{R L} h_{R}\left\{\Delta_{R L}-1 / 3\right\} C_{R}$ (we assume that $h_{R} \neq 0$ ). Under this assumption and taking into account the independence of functions $C_{R}$ we get

$$
\left[l_{R L}+\Gamma(R)\right] \varepsilon_{0}=0
$$

Therefore constants $l_{R L}$ do not depend on subscript $L$, i.e.

$$
\left[l_{R}+\Gamma(R)\right] \varepsilon_{0}=0
$$

This equation admits non-trivial solutions only for $\left|l_{R}\right|=1$. Note that substituting the relax harmonicity conditions in "no force" conditions one can find a relation $l_{R} h_{R}=\varsigma_{R}$ and the characteristic equation. Since $\left|l_{R}\right|=1$, we get $h_{R}^{2}=1, l_{R}=\varsigma_{R} h_{R}$. So under "no force" conditions the relax harmonic superposition rule coincides with the harmonic superposition rule and one can say that the requirement of the supersymmetry supports the harmonic superposition.

To guarantee that a configuration obtained as a result of intersecting of single branes is supersymmetric one has to study a compatibility conditions of all requirements (92). In order to analyse these requirements let us introduce $S$ as a special set of signs

$$
S=\{ \pm, \ldots, \pm\}, \quad \text { or } \quad S(R)= \pm
$$

We search a set $S$ which admits an existence of $\varepsilon_{0}$ such that

$$
P_{R}^{S(R)} \varepsilon_{0}=0
$$

for all $R$. Here $P_{R}^{S(R)}$ is a projector

$$
P_{R}^{S(R)}=\frac{1+S(R) \Gamma(R)}{2} .
$$

We will demonstrate, that one can find an appropriate $S$ and $\varepsilon_{0}$, if the incidence matrices satisfy the characteristic equations. In this case of $11 \mathrm{D}$ supergravity they lead to the restrictions on the incidence matrix

$$
\sum_{N=0}^{D-1} \Delta_{R N} \Delta_{R^{\prime} N}=1, \quad \text { or } \quad \sum_{N=0}^{D-1} \Delta_{R N} \Delta_{R^{\prime} N}=3, \quad R \neq R^{\prime} .
$$

If the incidence matrix satisfies these conditions one can check that

$$
\left[\Gamma(R), \Gamma\left(R^{\prime}\right)\right]=0
$$

Using (97) one can introduce new projection operators 


$$
P^{S}=\prod_{R} P_{R}^{S(R)}
$$

with the following properties:

$$
\begin{aligned}
& P^{S} P^{S^{\prime}}=\delta_{S S^{\prime}} P^{S}, \\
& \sum_{S} P^{S}=1 .
\end{aligned}
$$

Acting by $\sum_{S} P^{S}$ on an arbitrary spinor $\varepsilon_{i} \neq 0$ and using $(100)$, one can obtain the existence of the set $S_{0}$, such that $P^{S_{0}} \varepsilon_{i}=\varepsilon_{0} \neq 0$. Moreover for every $R P_{R}^{-S_{0}(R)} \varepsilon_{0}=0$, comparing this result with the equation (94) one can conclude, that $-\varsigma_{R} S_{0}(R)$ is supersymmetric signs set for $h_{R}$.

To summarize, using a simple algebraic method we have constructed the general D-dimensional intersecting p-brane solutions which satisfy the harmonic function superposition rule and possess $S$ - and $T$-dualities. The intersections of p-branes are controlled by the characteristic equations. These equations have solutions only for quantized values of scalar coupling parameters. Some of these solutions in the cases of $D=11$ and $D=10$ provide the metrics with regular horizons and non-zero entropy.

\section{ACKNOWLEDGMENTS}

We are grateful to I. V. Volovich for useful discussions. This work is partially supported by the RFFI grants 96-01-00608 (I.A. and O.R.) and 96-01-00312 (M.I.).

[1] K. S. Stelle, hep-th/9701088

[2] C. Hull and P. Townsend, Nucl. Phys. B438(1995)109

[3] E. Witten, Nucl. Phys. B443(1995)85

[4] P. Townsend, hep-th/9507048

[5] J. H. Schwarz, Phys. Lett. B367 (1996) 97-103, hep-th/9510086, hep-th/9607201

[6] A. Sen, Mod. Phys. Lett. A11 (1996) 827

[7] A. Strominger and C. Vafa, Phys. Lett. B379 (1996) 99-104, hep-th/9601029

[8] A. A. Tseytlin, Mod. Phys. Lett. A11 (1996) 689-714, hep-th/9601177

[9] C. Callan and J. Maldacena, Nucl. Phys. B472 (1996) 591-610, hep-th/9602043

[10] G. Papadopoulos and P. K. Townsend, Phys. Lett. B380 (1996) 273-279, hep-th/9603087

[11] A. A. Tseytlin, Nucl. Phys. B475 (1996) 149, hep-th/9604035

[12] G. Papadopoulos, Fortsch. Phys. 44 (1996) 573-584, hep-th/9604068

[13] I. R. Klebanov and A. A. Tseytlin, Nucl. Phys. B475 (1996) 179, hep-th/9604166

[14] K. Behrndt, E. Bergshoeff and B. Janssen, Phys. Rev. D55 (1997) 3785-3792, hep-th/9604168

[15] J. P. Gauntlett, D. A. Kastor and J. Traschen, Nucl. Phys B478 (1996) 544-560, hep-th/9604179

[16] E. Bergshoeff, R. Kallosh, T. Ortin, Nucl. Phys. B478 (1996) 156-180, hep-th/9605059

[17] A. A. Tseytlin, gr-qc/9608044

[18] E. Bergshoeff, C. M. Hull and T. Ortin, Nucl. Phys. B451 (1995) 547, hep-th/9504081

[19] G. T. Horowitz, gr-qc/9604051

[20] K. Behrndt, G. L. Cardoso, B. de Wit, R. Kallosh, D. Lust and T. Mohaupt, hep-th/9610105

[21] R. Emparan, hep-th/9610170

[22] C. G. Callan, Jr., S. S. Gubser, I. R. Klebanov and A. A. Tseytlin, hep-th/9610172

[23] E. Bergshoeff, M. De Roo and S. Panda, Phys. Lett. B390 (1997) 143-147, hep-th/9609056

[24] G. Papadopoulos and P. K. Townsend, Phys. Lett. B393 (1997) 59-64, hep-th/9609095

[25] A. A. Tseytlin, Nucl. Phys. B487 (1997) 141-154, hep-th/9609212

[26] E. Bergshoeff, M. de Roo, E. Eyras, B. Janssen and J. P. van der Schaar, hep-th/9612095

[27] R. Arguirio, F. Englert and L. Houart, hep-th/9701042

[28] A. Volovich, hep-th/9608095, Nucl. Phys. B492 (1997)

[29] I. Ya. Aref'eva, K. S. Viswanathan and I. V. Volovich, hep-th/9609225

[30] I. Ya. Aref'eva and A. I. Volovich, hep-th/9611026 
[31] V. D. Ivashchuk and V. N. Melnikov, hep-th/9612089

[32] I. Ya. Aref'eva and O. A. Rytchkov, hep-th/9612236

[33] I. Ya. Aref'eva, K. S. Viswanathan, A. I. Volovich and I. V. Volovich, hep-th/9701092

[34] I. Ya. Aref'eva, M. G. Ivanov and I. V. Volovich, hep-th/9702079

[35] N. Ohta, hep-th/9702164

[36] N. Hambli, hep-th/9703179

[37] A. Dubholkar, G. W. Gibbons, J. A. Harvey, F. Ruiz Ruiz, Nucl. Phys. 340 (1990) 33.

[38] S. D. Majumdar, Phys. Rev 72, 930 (1947); A. Papapetrou, Proc. R. Irish Acad. A51, 191 (1947)

[39] C. G. Callan, J. A. Harvey and A. Strominger, Nucl. Phys. B 359 (1991) 611 